\title{
Infantile scimitar syndrome with severe pulmonary hypertension: successful treatment with coil embolization of the systemic arterial supply to the sequestered lung
}

\author{
Jean-Pierre Pfammatter, Ingrid Luhmer, Hans Carlo Kallfelz \\ Division of Pediatric Cardiology, Children's Hospital, Hannover Medical Scbool Hannover, Germany
}

\begin{abstract}
Scimitar syndrome is a rare cardiopulmonary malformation. Presentation in infancy is usually associated with pulmonary hypertension and severe symptoms. We discuss treatment of such an infant. Two abnormal systemic vessels supplying the sequestered lower part of the right lung were embolized using catheter-inserted coils. The patent arterial duct was surgically ligated. These procedures resulted in a significant reduction of the shunt and the level of pulmonary hypertension, as well as in an impresssive improvement of symptoms.
\end{abstract}

Key words: Coil embolization, pulmonary hypertension, scimitar syndrome

$\mathrm{S}$ CIMITAR SYNDROME IS A WELL DESCRIBED entity, characterised by partially anomalous connection of the pulmonary veins to the inferior caval vein, hypoplasia of the right lung, right-sided position of the heart and systemic arterial supply from the abdominal aorta to the sequestered segments of the lung, usually in the lower lobe of the right lung. Presentation in the neonatal period or in infancy is rare, but then symptoms are usually severe with the prognosis being related to the associated cardiovascular anomalies. In older children, symptoms are less severe and the syndrome is usually detected when investigation is undertaken for recurrent pulmonary infections, chest $\mathrm{x}$-ray abnormalities or a heart murmur. We discuss presentation of the syndrome in a neonate with severe symptoms. Coil embolization of the abnormal systemic arterial supply to the sequestered pulmonary segments, together with surgical closure of the arterial duct resulted in a favourable outcome.

Correspondence to: Dr Jean-Pierre Pfammatter, MD. Division of Pediatric Cardıology Chıldren's Hospital, Freiburgstrasse, $\mathrm{CH}-3010$ Berne, Switzerland. Fax: ++41316329748

Accepted for publication 7 June 1996

\section{Case report}

This boy was born after an uneventful pregnancy at 40 weeks of gestation as the second child to healthy parents. Family history did not reveal cardiopulmonary anomalies, except for frequent recurrence of pulmonary airway obstructions necessitating inhalative bronchodilatatory treatment in the older brother. In that brother, a rightsided heart was found as an incidental finding later on. He underwent cardiac catheterization and cardiovascular malformations could be excluded.

The patient reported was found to have a rightsided heart at a routine examination at three days of age. Investigation in another hospital led to the suspicion of scimitar syndrome, and the patient was sent to our centre for evaluation at the age of three weeks. On admission, the boy was tachypneic with almost silent pulmonary auscultation on the right side in comparison to normal auscultatory findings on the left side. Heart sounds were loudest at the right sternal border. There was a single and loud second heart sound, as well as a grade II/VI systolic murmur. Heart rate was 166/minute. The liver was enlarged. Chest X-ray showed deviation of the heart and the mediastinum to the right side of the thorax, severe 


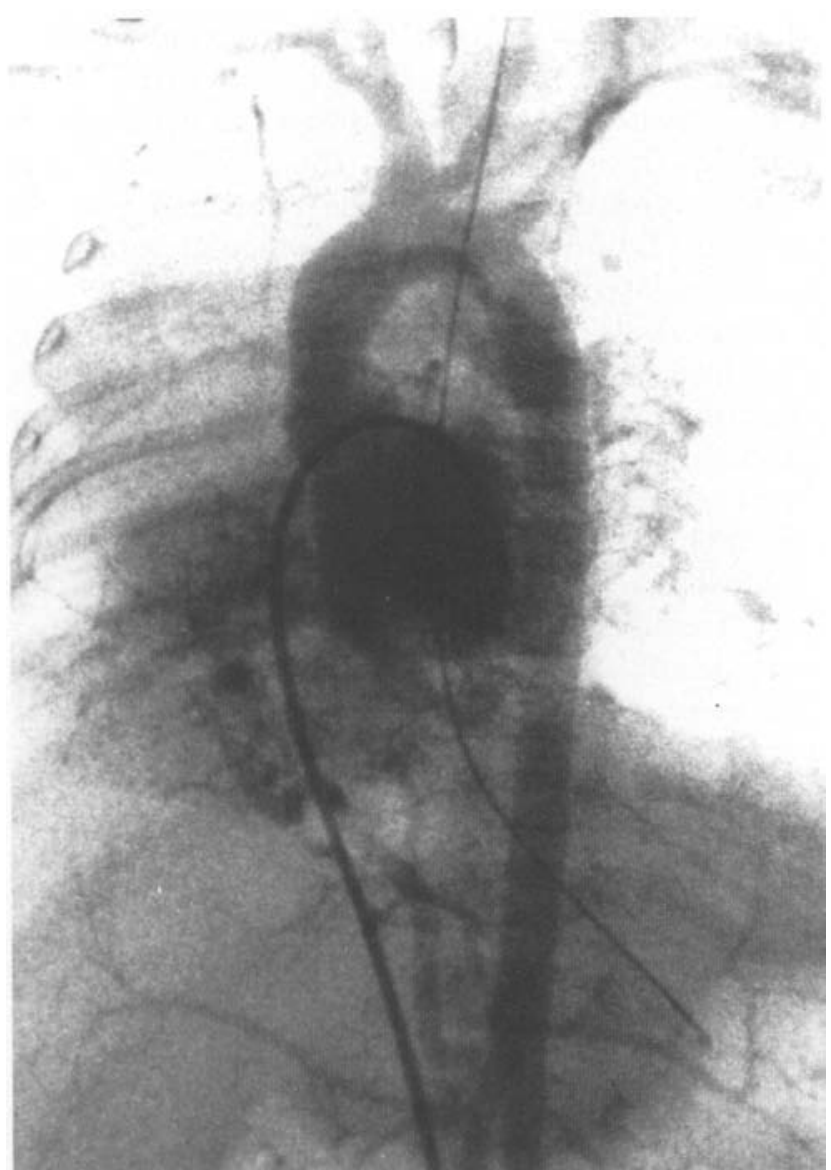

Figure 1.

Two systemic vessels originating from the abdominal aorta and supplying a large area of the right lung are seen: the proximal vessel arises at the level of the diaphragm, the more distal vessel arises near the celiac artery. hypoplasia of the right lung, and compensatory expansion of the left lung. Vascular markings on the left side were increased. The electrocardiogram showed prominent $\mathrm{P}$-waves in the right precordial leads and signs of right ventricular hypertrophy. Echocardiography showed usual atrial arrangement, an atrial septal defect with left-to-right shunt, patency of the arterial duct with a small left-to-right shunt, an enlarged right atrium and right ventricular hypertrophy. The left-sided pulmonary veins were shown to drain into the left atrium, whereas the right-sided pulmonary veins connected into the inferior caval vein. There was evidence of severe pulmonary hypertension. Pulmonary perfusion scintigraphy revealed a perfusion imbalance, with a left/right ratio of 85/15\%.

Cardiac catheterization was performed at the age of 6 weeks. The standard femoral venous approach was used, with the patient under general anesthesia. Oxygen saturation in the superior caval vein was $58 \%$, in the right atrium $83 \%$, and in the inferior caval vein $91 \%$. Systolic pulmonary pressure was at systemic level $(67 \mathrm{mmHg}$, with the systolic left ventricular pressure being $70 \mathrm{mmHg}$ at the same time). The left-to-right shunt at atrial level was calculated to be $56 \%$, and the pulmonary resistance was elevated to 4 Woods units. Angiography revealed an atrial septal defect, patency of the arterial duct, right-sided heart, partially anomalous pulmonary venous connection from the entire right lung via a vertically descending (scimitar) vein into the inferior caval vein just below the right atrium. Additionally, two abnormal arteries originating from the abdominal aorta were identified supplying a large sequestered area in the lower part of the right lung (Fig. 1). As these arteries were considered to add significantly to the total left-to-right shunting and to pulmonary hypertension, it was decided to occlude them. The distal vessel (diameter $2.5 \mathrm{~mm}$ )

Table 1: Comparison of catheterization data

\begin{tabular}{lccc}
\hline $\begin{array}{c}\text { Parameters at } \\
\text { catheterization }\end{array}$ & Pre-embolization & Post-embolization & $\begin{array}{c}\mathbf{8} \text { months } \\
\text { after embolization }\end{array}$ \\
\hline Pulmonary arterial pressure & $67 / 19$ (mean 37) & $52 / 22$ (mean 31) & $40 / 12($ mean 25) \\
Systemic pressure & $70 / 23$ (mean 38) & $76 / 34$ (mean 42) & $96 / 59($ mean 73$)$ \\
Ratio pulmonary/systemic pressure & 1 & 0.7 & 0.4 \\
PVR (Woods units) & 4 & not assessed & 2 \\
Left-right shunt & $56 \%$ & not assessed & $36 \%$ \\
\hline
\end{tabular}

$(P V R=$ pulmonary vascular resıstance, $L V=$ left ventricle $)$ 
was occluded first using two steel coils with a helical diameter of $3 \mathrm{~mm}$ (MWCE 38-4-3, Cook Europe Inc). The more proximal vessel (diameter $3,5 \mathrm{~mm}$ ) was then occluded with three coils, the first two having a coiled diameter of $5 \mathrm{~mm}$ (MWCE 38-5-5) and the last one having a diameter of $3 \mathrm{~mm}$. Angiography after 15 minutes revealed complete occlusion of both vessels with no residual flow of contrast (Fig. 2). Systolic pulmonary arterial pressure had dropped to about $3 / 4$ of systemic pressure (Table 1 ). The boy was left intubated and ventilated, and treatment with prostacyclin (epoprostenol) infusion was started in order further to decrease pulmonary resistance to allow surgical ligation of the duct. This was performed without complications 6 days after the interventional catheterization. The patient recovered well but needed further treatment consisting of oral and inhalative bronchodilatatory agents for frequently recurrent pulmonary airway obstruction.

Repeat catheterization was performed at the age of 9 months. The child was still on bronchodilatatory treatment, but otherwise had thrived well and had no symptoms except for a slightly

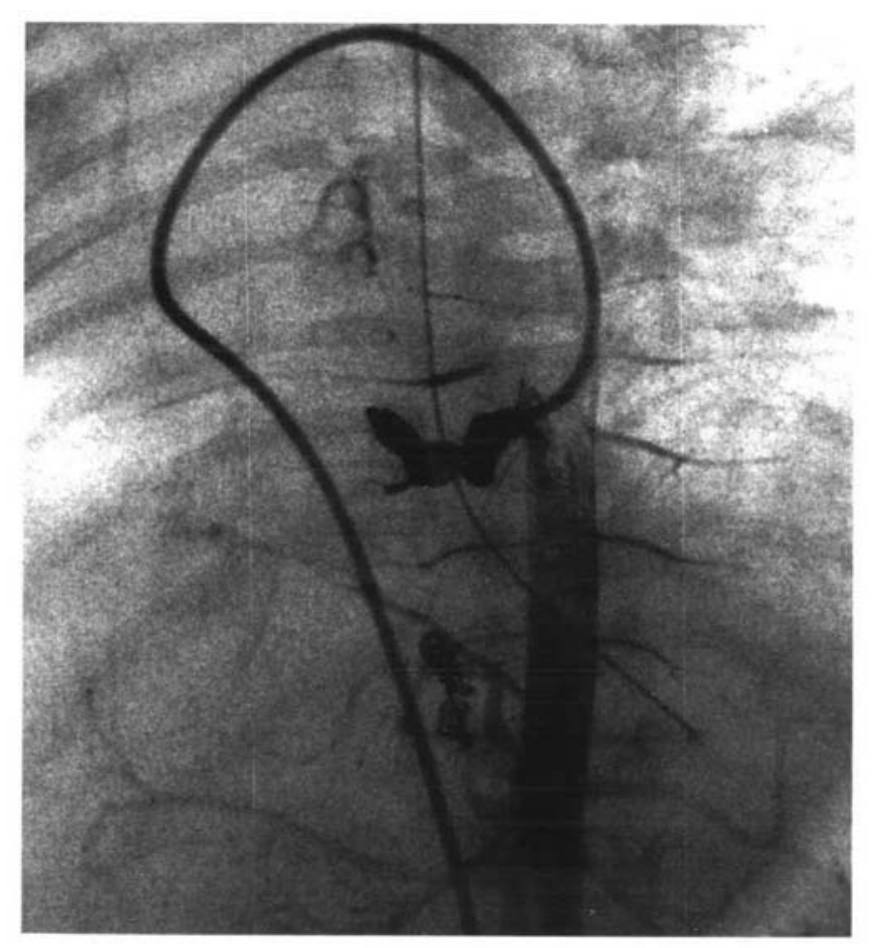

Figure 2.

Selective arteriograpby of the more proximal of the two abnormal systemic vessels shows no residual flow after placement of the coils. More distally, the second artery is shown with the couls already in place. No flow of contrast is seen. elevated respiration rate. Catheterization revealed residual left-to-right shunting at atrial level (36\%). Systolic pulmonary arterial pressure was 40 $\mathrm{mmHg}$ (mean 24), with the left ventricular systolic pressure at the same time being at 96 $\mathrm{mmHg}$ (Table 1). Pulmonary vascular resistance was calculated to be 2 Woods units.

In the lack of severe symptoms, and because of the clinically stable condition, no further invasive treatment was deemed necessary. At the last follow-up visit (March 1995, 19 months after the intervention), the child was in good health. There was no history of pulmonary infections, the patient was still on prophylactic inhalative bronchodilatatory treatment but he had suffered no further episodes of acute pulmonary airway obstructions. The electrocardiogram showed signs of mild right ventricular hypertrophy. Follow-up examinations are planned at yearly intervals.

\section{Discussion}

Scimitar syndrome is a distinct cardiovascular and pulmonary malformation. ${ }^{2}$ Clinically, there are two modes of presentation: in the older child the syndrome usually manifests as recurrent pulmonary infections or as a heart murmur. In these patients impressive symptoms are rarely found, and prognosis is generally good. ${ }^{3}$ On the other hand, the variant encountered in infancy is associated with marked pulmonary hypertension ${ }^{4}$ and is usually associated with severe symptoms of respiratory distress, heart failure, or even cyanosis. In combination with other cardiovascular malformations, the prognosis is poor even when surgical or interventional therapy is undertaken. 4,5

Symptomatic infants with the syndrome are characterised by the presence of usually severe pulmonary hypertension with systemic or suprasystemic pulmonary arterial pressures. ${ }^{5}$ Pulmonary hypertension is found in these patients in the presence of small shunts which, in other circumstances, do not cause pulmonary hypertension. ${ }^{3}$ It has been hypothesized that the abnormal systemic flow to the sequestered area of the right lower lung, which is typically found in the infantile form of the syndrome, contributed significantly to the severity of symptoms and to pulmonary hypertension. ${ }^{4,} 6$ Histologic examination of the pulmonary vascular structures in infants with scimitar syndrome revealed an abnormally increased muscularity of pre- and intraacinar pulmonary arteries in both lungs. ${ }^{7}$

Treatment of the scimitar syndrome, when seen in infancy, is directed at reducing the shunt and 
decreasing pulmonary hypertension. This seems to be best achieved by interruption of the abnormal systemic arterial supply from the abdominal aorta to the sequestered segments of the right lung, together with closure of the usually associated shunt lesions, such as an atrial septal defect or a patent arterial duct. Standard therapy has been surgical. This treatment is associated with considerable morbidity and mortality. ${ }^{5}$ Lobectomy and pneumonectomy have also been performed. ${ }^{3,4}$ Advances in interventional catheterization have now made it possible effectively to occlude abnormal intrathoracic vessels. ${ }^{8}$ As early as in 1982, this method was advocated for the treatment of scimitar syndrome in infancy. In this early experience embolization using Gelfoam fragments was unsuccessfully attempted in two infants but was successful in one. ${ }^{6}$ To the best of our knowledge, only four infants with scimitar syndrome who have undergone successful embolization have been reported so far. One large experience on the use of coil embolization in children included one child with scimitar syndrome in whom successful embolization had been performed but the age of the patient was not specified.? In another study, embolization was attempted in four infants with scimitar syndrome (using coils in two cases, a detachable balloon and tissue adhesive in one patient, respectively) and was successful in two. ${ }^{5}$

In our patient, the embolization of the abnormal vessels supplying a large sequestered area in the lower lobe of the right lung resulted in a marked reduction of pulmonary arterial pressure and in a significant reduction of the shunt volume. The contribution of surgical ligation of the duct is difficult to assess. Based on the experience of others, ${ }^{4}$ it might well have been of minor importance. The favorable outcome shows that early interruption of systemic flow through pulmonary sequestrations is a promising therapeu- tic option to decrease the shunt volume and ameliorate pulmonary hypertension. Transcatheter embolization cannot be compared to results of surgical ligation, as only very few infants with the former approach have been reported. Our experience, nonetheless, shows that coil embolization can be successfully carried out even in small and sick infants. It should probably be considered as the optimal mode of treatment in infants with symptomatic scimitar syndrome. As with all interventions in this disease, it remains palliative in nature.

\section{References}

1. Fisher DR, Zuberbühler JR. Pulmonary venous abnormalıties. In: Anderson RH, Macartney FJ, Shinebourne EA, Tynan M. Pediatric Cardiology. Churchill Livingston, Edinburgh 1990; vol I, p.509

2. Neill CA, Ferencz C, Sabiston DC, Sheldon H. The familial occurrence of hyppoplastic right lung with systemic arterial supply and venous drainage Scimitar syndrome. Bulletin of the J Hopkins Hospital 1960; 107: 1-21

3. Canter CE, Martin TC, Spray TL, Weldon CS, Strauss AW. Scimitar syndrome in infancy. Am J Cardiol 1986; 58 852-854

4. Dupuis C, Charaf LAC, Breviere GM, Abou P. Infantile form of the scimitar syndrome with pulmonary hypertension. Am J Cardiol 1993; 71: 326-330

5. Gao YA, Burrows PE, Benson LE, Rabınovitch M, Freedom RM. Scimıtar syndrome in infancy. J Am Coll Cardiol 1993; 22: 873-882

6. Dickinson DF, Galloway RW, Massey R, Sankey R, Arnold R Scimitar syndrome in infancy. Role of embolization of systemic arterial supply to right lung. Br Heart J 1982; 47 : 468-472

7. Haworth SG, Sauer U, Bühlmeyer K. Pulmonary hypertension in scimitar syndrome in infancy. $\mathrm{Br}$ Heart $\mathrm{J}$ 1983; 50: 182-189

8. Reidy JF, Jones ODH, Tynan M, Baker EJ, Joseph MC Embolisation procedures in congenital heart disease. $\mathrm{Br}$ Heart J 1985; 54: 184-192

9. Perry SB, Radtke W, Fellows KE, Keane JF, Lock JE. Coll embolization to occlude aortopulmonary collateral vessels and shunts in patients with congenital heart disease. $\mathrm{J}$ Am Coll Cardiol 1989; 13: 100-108 